\title{
Psychogenic nonepileptic seizures; beyond differentiating from epileptic seizures
}

\author{
Sang Ook Nam, MD, PhD \\ Department of Pediatrics, Pusan National University Children's Hospital, Yangsan, Korea
}

Psychogenic nonepileptic seizures (PNES) is a paroxysmal disorder with a variety of sensory, motor, behavioral, and consciousness alterations that appears similar to epileptic seizures but lacks epileptic activity in the brain. PNES is commonly mistaken for intractable epilepsy and treated inappropriately with anticonvulsants. The paroxysmal events of PNES include physical symptoms originating from psychological problems, although patients are more concerned and interested in their physical symptoms than their psychological problems because they do not understand that a psychological stressor can cause physical symptoms. Patients often have difficulty accepting the diagnosis and often reject the fact that PNES is due to psychological rather than physical problems.

Posttraumatic PNES is common in adults because prior trauma, including physical and sexual abuse, affects $75 \%$ of adult patients with PNES. ${ }^{1)}$ In contrast, developmental PNES due to coping difficulties is more prevalent in children because everyday stressors, such as family discord, school problem, interpersonal problems, and medical problems are more common than physical or sexual abuse. ${ }^{2)}$

A detailed history, seizure semiology, and conventional and video electroencephalography (EEG) findings are helpful for differentiating between PNES and epileptic seizures. In personality tests, such as the Personality Assessment Inventory and the Minnesota Multiphasic Personality Inventory-2, patients with PNES showed higher scores on the conversion, hypochondriasis, and hysteria subscales than patients with epileptic seizure. Elevation of the serum prolactin level to more than twice normal in the blood drawn at 10-20 minutes after a seizure may help confirm the diagnosis of epileptic seizures rather than PNES.3)

Ictal semiology also helps with the differential diagnosis. Long duration, fluctuating course, asynchronous or side-to-side movements, ictal eye closure at onset, ictal crying, modulation of event intensity by a bystander, and postictal memory recall favor PNES. ${ }^{4)}$ The likelihood of an epileptic seizure is high when the events include an abrupt onset, ictal eye-opening, eye deviation, and postictal confusion or sleep. When "the rule of $2 \mathrm{~s}$ " is met: (1) PNES at least twice a week; (2) refractory to two anticonvulsants; and (3) at least 2 EEG without epileptiform discharge, positive predictive value of PNES is as high as $85 \%$ in patients with PNES confirmed on video EEG. ${ }^{5)}$ The video EEG finding of a lack of an ictal EEG change during a seizure is the gold standard for confirming the diagnosis of PNES.

To facilitate patient acceptance and efficient treatment, both doctors and patients must understand that the continued psychologic stress leads to functional and structural abnormalities in the brain and neuroendocrine system, causing the uncontrollable paroxysmal events of PNES.6) Electrophysiological, structural, and functional brain imaging studies using EEG, magnetic resonance imaging, functional magnetic resonance imaging, and positron emission tomography have been used since the 2010s to reveal evidence of functional, structural, and connectivity abnormalities in the execution (inferior frontal gyrus, parietal cortex), emotion (anterior cingulate cortex, insula), and sensorimotor (supplementary motor, pre- and postcentral cortex) areas in patients with PNES.7) One study reported decreased connectivity between the cortical and subcortical regions of the basal ganglia and interhemispheric connectivity in patients with PNES. ${ }^{8)}$ These findings indicate that the brains of patients with PNES can exhibit a paroxysmal attack of emotion, movement, and behavior due to abnormal interactions between areas involving emotional and cognitive control, sensorimotor activity, and involuntary motor activity.

To ensure proper treatment, it is necessary to make an accurate diagnosis by using information from diverse sources, including video EEG, and then convey the diagnosis and its mechanism to patients and their families using a warm but clear attitude. This is the first and most important stage of treatment in all patients with PNES that can make the symptoms stop or greatly improve if the disease duration is not prolonged and the psychological conflict is not severe. The event reduction outcomes are better with immediate than delayed anticonvulsant withdrawal. ${ }^{9)}$

Psychiatric collaboration is necessary because patients with PNES often have psychiatric comorbidities, such as anxiety, depression, posttraumatic stress disorder, attention deficit hyperactivity disorder, and somatic symptom disorder. ${ }^{5)}$ Significant

Corresponding author: Sang Ook Nam, MD, PhD. Department of Pediatrics, Pusan National University Children's Hospital, 20 Geumo-ro, Mulgeum-eup, Yangsan 50612, Korea

® E-mail: neuroped@naver.com, https://orcid.org/0000-0003-1312-3483

Received: 14 July, 2020, Revised: 21 August, 2020, Accepted: 27 August, 2020

This is an open-access article distributed under the terms of the Creative Commons Attribution Non-Commercial License (http://creativecommons.org/licenses/bync/4.0/) which permits unrestricted non-commercial use, distribution, and reproduction in any medium, provided the original work is properly cited.

Copyright (c) 2021 by The Korean Pediatric Society 
advances in evidence-based management have also been made in the psychiatric treatment of PNES during the past decade. In randomized controlled trials, cognitive behavioral therapy (CBT) alone or with a selective serotonin reuptake inhibitor like sertraline showed significantly improved seizure reduction compared with standard medical care, whereas standard medical care or sertraline alone was ineffective. ${ }^{10)} \mathrm{CBT}$ also improved patient depression, anxiety, quality of life, and global functioning. However, the event is less likely to be free in women, those with anxiety/depressive disorder, and patients with social security benefits, many of whom develop other types of medically unexplained functional neurologic symptoms. ${ }^{11)}$

Neurologists must actively intervene and cooperate with a multidisciplinary team that includes patients, families, psychiatrists, psychologists, and social workers in the process of referring patients to mental health professionals.

Conflicts of interest

No potential conflict of interest relevant to this article was reported.

\section{Acknowledgments}

This study was supported by a 2020 research grant from Pusan National University Yangsan Hospital.

See the article "Myths and truths about pediatric psychogenic non-epileptic seizures" via https://doi.org/10.3345/cep.2020. 00892

\section{References}

1. Duncan R, Oto M. Predictors of antecedent factors in psychogenic nonepileptic attacks: multivariate analysis. Neurology 2008;71:1000-5.

2. Plioplys S, Doss J, Siddarth P, Bursch B, Falcone T, Forgey M, et al. A multisite controlled study of risk factors in pediatric psychogenic nonepileptic seizures. Epilepsia 2014;55:1739-47.

3. LaFrance WC Jr. Baker GA, Duncan R, Goldstein LH, Reuber M. Minimum requirements for the diagnosis of psychogenic nonepileptic seizures: a staged approach: a report from the International League Against Epilepsy Nonepileptic Seizures Task Force. Epilepsia 2013;54: 2005-18.

4. Devinsky O, Gazzola D, LaFrance WC Jr. Differentiating between nonepileptic and epileptic seizures. Nat Rev Neurol 2011;7:210-20.

5. Davis BJ. Predicting nonepileptic seizures utilizing seizure frequency, EEG, and response to medication. Eur Neurol 2004;51:153-6.

6. Yeom J, Bernard H, Koh S. Myths and truths about pediatric psychogenic nonepileptic seizures. Clin Exp Pediatr 2020. https://doi.org/10.3345/ cep.2020.00892

7. Perez DL, Dworetzky BA, Dickerson BC, Leung L, Cohn R, Baslet G, et al. An integrative neurocircuit perspective on psychogenic nonepileptic seizures and functional movement disorders: neural functional unawareness. Clin EEG Neurosci 2015;46:4-15.

8. Barzegaran E, Carmeli C, Rossetti AO, Frackowiak RS, Knyazeva MG. Weakened functional connectivity in patients with psychogenic nonepileptic seizures (PNES) converges on basal ganglia. J Neurol Neurosurg Psychiatry 2016;87:332-7.

9. Oto M, Espie CA, Duncan R. An exploratory randomized controlled trial of immediate versus delayed withdrawal of antiepileptic drugs in patients with psychogenic nonepileptic attacks (PNEAs). Epilepsia 2010;51:19949.

10. LaFrance WC Jr. Reuber M, Goldstein LH. Management of psychogenic nonepileptic seizures. Epilepsia 2013;54(Suppl 1):53-67.

11. McKenzie P, Oto M, Russell A, Pelosi A, Duncan R. Early outcomes and predictors in 260 patients with psychogenic nonepileptic attacks. Neuro$\log 2010 ; 74: 64-9$.

How to cite this article: Nam SO. Psychogenic nonepileptic seizures; beyond differentiating from epileptic seizures. Clin Exp Pediatr 2021;64:282-3. https://doi.org/10.3345/cep.2020.01207 